\title{
Live E! Project: establishment of infrastructure sharing environmental information
}

\author{
Satoshi Matsuura $\dagger$ \\ sato-mat@is.naist.jp \\ Shinichi Doi $\dagger$ \\ shini-do@is.naist.jp
}

\author{
Hiroki Ishizuka ††† \\ isi@unl.im.dendai.ac.jp \\ Shinichi Ishida $\dagger$ \\ ishida@hongo.wide.ad.jp
}

\author{
Hideya Ochiai $\nmid \dagger$ \\ jo2lxq@hongo.wide.ad.jp \\ Masaya Nakayama †† \\ nakayama@nc.u-tokyo.ac.jp
}

\author{
Hiroshi Esaki†† \\ hiroshi@wide.ad.jp \\ Hideki Sunahara $†$ \\ suna@wide.ad.jp
}

†Graduate School of Information Science, Nara Institute of Science and Technology

8916-5 Takayama-cho, Ikoma-shi, Nara 630-0192, Japan

$\dagger †$ The University of Tokyo, 7-3-1 Hongo, Bunkyo-ku, Tokyo, 113-8656, Japan

$\dagger \dagger \dagger$ Department of Information Systems and Multimedia Design School of Engineering, Tokyo Denki University 2-2 Kanda-Nishiki-Cho Chiyoda-ku, Tokyo 101-8457,Japan

\begin{abstract}
The Live E! project is an open research consortium among industry and academia to explore the platform to share the digital information related with the earth and our living environment. We have getting a lot of low cost sensor nodes with Internet connectivity. The deployment of broadband and ubiquitous networks will enable autonomous and global digital information sharing over the globe. In this paper, we describe the technical and operational overview of Live E! project, while discussing the objective, such as education, disaster protection/reduction/recovery or business cases, and goal of this project activity.
\end{abstract}

\section{Introduction}

Recent natural disaster, e.g., hurricane or global warming, or heat island effect in the metropolitan let increase the attention and interesting on grasping the detailed status of space. This is because, due to these disasters, our social life and business activity could be seriously degraded or sometimes be damaged. When we could realize the relationship of cause and effect, it would be expected to reduce and protect the damage by these disasters. The structure change of metropolitans in the developed countries and rapid inflation of cities in Asian countries make complex and difficult to realize the real status and tendency of global weather system.
We have getting a lot of low cost sensor nodes with Internet connectivity. The deployment of broadband and ubiquitous networks will enable autonomous and global digital information sharing over the globe, using these sensor nodes. When these wide variety of sensor nodes are autonomously connected and the sensor information let available to all the node on the Internet space, different types of sensor information, e.g., video or still image captured by Web camera, temperature, location, IR-image or chemical, can be integrated for data analysis. Then, we will be able to create so wide variety of applications and possibilities. When these sensor nodes are connected with broadband Internet, these information can be available even in real-time fashion. We have realized and proposed the activity, called Live E! Project [1], that is a sensor networks sharing all the digital information related with the at large status of the Earth for any purpose and for anyone.

In this paper, we describes the technical and operational overview of Live E! project, while discussing it's objective, such as education, disaster protection/reduction/recovery or business cases, and goal of this project activity.

\section{Live E! Project}

\subsection{Overview of Live E! Project}

Live E! Project is a R\&D consortium founded by WIDE project and IPv6 Promotion Council, Japan. This project is aiming to establish the platform to share all digital informa- 


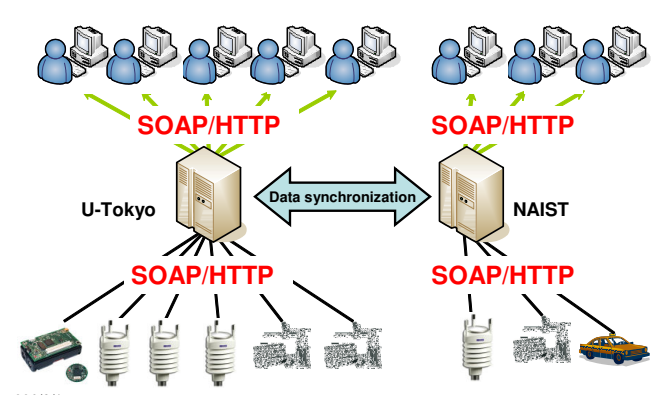

Figure 1. integrate interface by SOAP/HTTP

tion, generated by sensor devices all over the world. Now, these devices are installed and operated individually by each organization.

The information should be related with the live environmental information of the Earth. By sharing this digital information, we will be able to create new applications, which can contribute to safety and effective space (environment). Some applications, such as basic information for the protection of environment (e.g., a heat-island phenomena in metropolitan), educational material, public service, public safety or business applications, could use the common digital information for different purposes. Figure 1 shows the overview architecture of Live E! project. The working agendas of Live E! are installing a large number of sensors, combining every organization and individual to preserve global environment, and contributing environmental problems, education and businesses.

\subsection{Live E! deployment}

We have realized that the weather station with Internet connectivity has the following three application areas with single device.

(1) For Educational Material

Weather information data is useful data for education and for research on geophysics. There are wide variety of educational program on geophysics, related with the weather system, from the elementary school to the college. Actually, in Live E! project, we have worked on the educational program in the elementary school in Minato-ku in Metropolitan Tokyo, and on the engineering program in some high schools collaborating with university in Hiroshima.

(2) For Public Service, e.g., disaster reduction/recovery Weather information is very important and critical information for the case of disaster. In these days, we have a lot of natural disasters, such as flooding or earth-quake. Grasping the detailed information for the

\begin{tabular}{c|c}
\hline \multicolumn{2}{c}{} \\
\hline name & unit \\
\hline Temperature & centigrade \\
Humidity & $\%$ \\
Pressure & $\mathrm{hPa}$ \\
RainFall & $\mathrm{mm} / \mathrm{h}$ \\
WindSpeed & $\mathrm{m} / \mathrm{s}$ \\
WindDir & degree \\
\hline
\end{tabular}

\begin{tabular}{c|c}
\hline name & explanation \\
\hline address & address of sensor \\
location & installation location \\
ipAddr & IPv4 address \\
ip6Addr & IPv6 address \\
latitude & latitude of sensor \\
longitude & longitude of sensor \\
altitude & altitude of sensor \\
\hline
\end{tabular}

Table 1. unit \& examples of profile data

disaster case is useful for proactive and reactive program. These are disaster protection, reduction, and recovery. For example, the detailed weather information on the road or at the evacuation sites, the people could take appropriate evacuation path. Also, none knows the exact and detailed data on the heat-island effect at metropolitan. We must grasp the real status of town with large number of weather sensors.

(3) For Business case

There are a lot of potential business applications, by the use of weather information. One of example would be effective taxi dispatching using the rainfall information. Dispatching the taxi cabs around the area, getting the rain, leads to higher income by the increase of customer. The other example would be electricity power company. Once they can operate total energy control and management system, they could reduce the amount of investments on the power generator or power supplying system, which is very expensive facility for them. Seriously, they may start to think about total portfolio for business investments.

\subsection{Data normalization and access method}

In order to share sensing data with every organization and individual, first we need to normalize data format and access method. In this sub-section we introduce our data format with some examples.

The weather information is expressed by XML and transferred among the servers and clients using the SOAP. The sensor is not defined by node, but is defined by each sensor function. A typical internet weather station has multiple sensors in a single station in it, which sensors are temperature, humidity, pressure, rainfall, wind-speed and winddirection. This typical weather station has 5 objectives defined by XML. Each objective has their own profile information, such as location, sensor type, IP address or name.

As shown in Table 1, we basically adopt MKS-system on our platform. If a new kind of sensor is installed, project member will define its name and the unit (recently, we installed $\mathrm{CO} 2$ sensors, illumination sensors, acceleration sensors and other sensors). By SOAP web-services, we create a 


\begin{tabular}{l}
\hline getCurrentDataAll() \\
- get current data from all sensors \\
\hline getCurrentDataByAreaRect( $\mathrm{x} 1, \mathrm{y} 1, \mathrm{x} 2, \mathrm{y} 2$ ) \\
- get data generated in paticular region \\
\hline getDataByTimespan( sensorID, startTime, endTime ) \\
- get data by time-span \\
\hline getCurrentDataByType( sensorType ) \\
- get current data of specific sensor type \\
\hline getProfileAll() \\
- get all profile data \\
\hline getProfileByType( sensorType ) \\
- get profile data of specific sensor type \\
\hline putData( xmlDocument ) \\
- put sensing data \\
\hline setProfile( xmlDocument ) \\
- set profile data
\end{tabular}

Table 2. examples of SOAP web-services

user interface (Figure 1). As databases aresynchronized between two locaitons, users can select web services which they want to use.

As the table 1 shows, we also provide attribute information of sensors called "profile data". We normalize and provide sensing data and profile data as XML document. Application creators freely combine sensing data with profile data to meet each requirement.

Example web-services we prepared are shown in Table 2. Here, we introduce an example way to retrieve sensor data. The most popular web-service is "getCurrentDataAll()". By this function, user gets current data generated by all sensors. Following Perl script is an example code to do it.Of course, any language and operating system can access to sensor data by using SOAP.

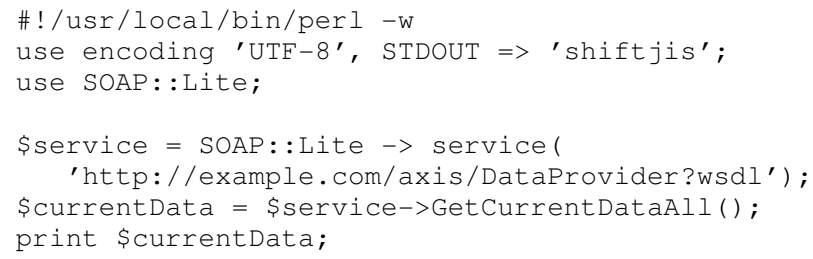

Figure 2 shows the result XML documnets when above example Perl script executed. We standardized XML format. Using other SOAP web-services, user always receives data as same XML format. Defining the name of webservices and XML format contributes to create application easily. In addition, as the above XML documents shows, we define a sensor-group. Sensor-group organizes individual sensors. In above XML document, class of sensor-group is "combinedSensor". This sensor-group organizes a multiple sensor as one of sensors. We define a new class of sensorgroup for several purposes. For example, class of "zipCode" is to manage a particular area, and class of "highAccuracy" is to pick up high performance sensors. Of course, we cre-

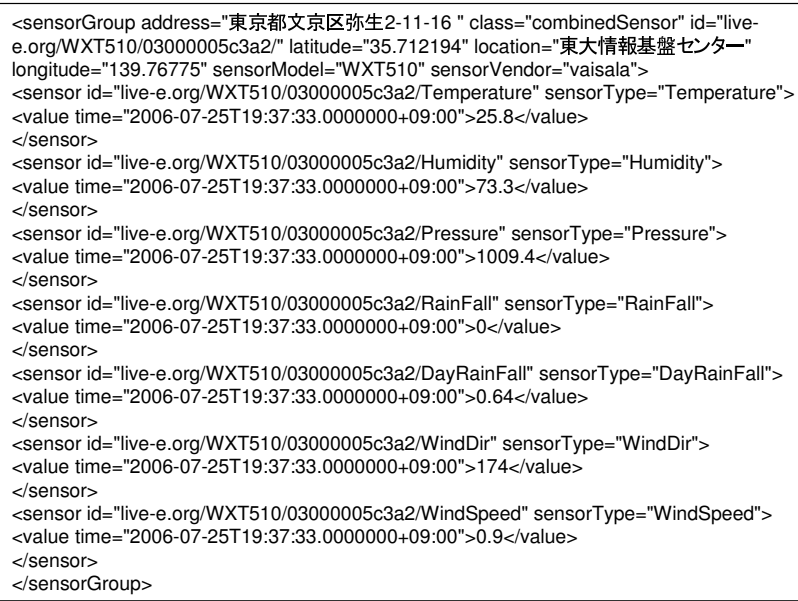

Figure 2. example of reply as XML document

ate new web-services by combining several classes. Sensorgroup improves a creativeness of raw sensing data.

Here, we introduce some of our applications created by SOAP web-services. Figure 3 shows the example of data displaying. On the web site, user can search each place for detailed data, which are graph of sensors, XML (or CVS) data and pictures of web cameras. And, with Google Earth ,we show the status of clouds and path information of a typhoon with sensor data. Live E! Project doesn't observe cloud and typhoon. Collaborating with other organizations, our project provides these data. We enhance the collaboration with others.

We also provide educational materials. We made a infomation viewer displaying real time sensor data. Students of elementary school constantly check and record the data of this viewer. Students will plot a graph with these data and discuss the difference of daily weather and local climate, and compare climate of home town with other place. Students will take interest in science with such actual experiences.

We have installed more than 100 stations and let them on-line. Some of stations have installed in Philippine by the collaboration with ASTI, or have installed in Thailand by the collaboration with PSU. For dense installation of weather sensors, we collaborate with Minato-ku in metropolitan Tokyo. Several sensors are installed at some elementary schools at Minato-ku and Kurashiki-city in Okayama. We will install about 30 stations in this fiscal year of 2006. By this installation, Minato-ku can have about $2 \mathrm{~km}$ mesh weather station network. And Kurashiki-city is focusing on the disaster protection and reduction against the flooding due to heavy rain. 


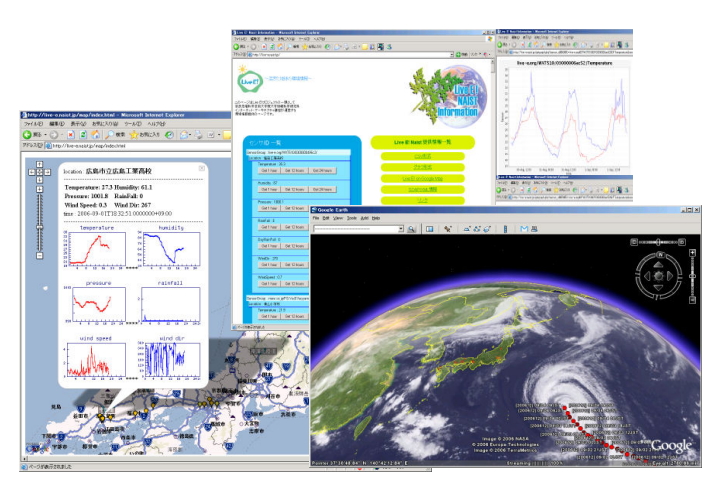

Figure 3. data displaying

\subsection{Future enhancement of Live E!}

- XML based Publish/Subscribe system

The more sensor devices and users increase, the more data and queries should be processed. The most popular web-service is "getCurrentDataAll()". Users are very interested in real time data. In order to provide this web-service stable, we create XML based PublishSubscribe system. This system don't store archived data but only provide current data. If users register for subscribing data, they constantly receive current data of all sensors from one of XML router. From now, we will try to set up of an application layer multicast based on user requirements to provide all web-services stable.

- Integration with InternetCAR system WIDE project has long time worked on the R\&D activity, where automobiles connect to the Internet. Automobiles can be realized as the mobile sensor node, running on the surface of the earth. We are now integrating the "InternetCAR" into our platform [2]. In addition, we are trying to use ad-hoc network for sensors not having the connectivity to the internet. Patrol nodes (ex. Post office cars, garbage trucks or buses) gather the sensing data via ad-hoc network [3].

- Support small nodes

There are lots of microcomputers not having the ability to translate XML document. In order to integrate lots of data created by microcomputers generate, we study about XML translational machinery [4].

- Scalable and autonomous data collection system The current system is a kind of client-server system. This is just fine for small scale system. However, the system must come up with the increase of sensors and the increase of type of sensors. Distributed and autonomous management and operation is mandatory.

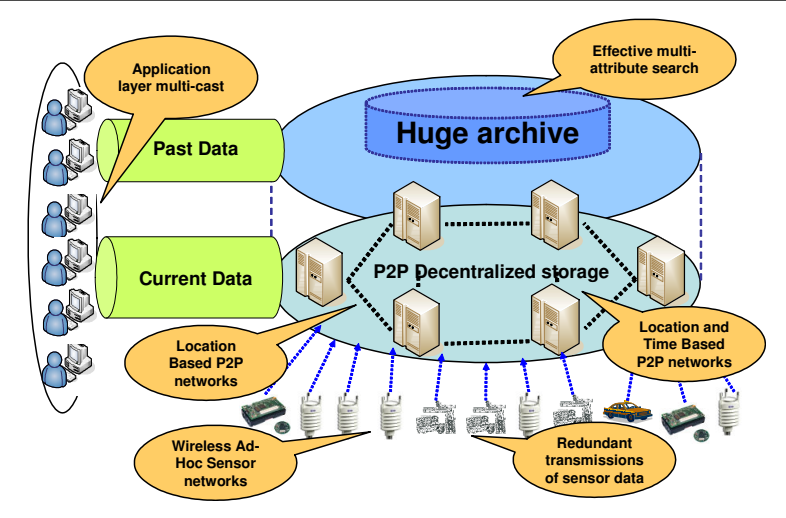

Figure 4. next Live E! architecture

We are studying about geographical location based peer-to-peer network [5].

As shown in Figure 4, next phase we focus on scarable and autonomous data collection and provision. We are evaluating the requirements of future sensor network, and integrate several kinds of researches.

\section{Conclusion}

The Live E! project is an open research consortium among industry and academia to explore the platform to share the digital information related with the earth and our living environment. Using the low cost weather sensor nodes with Internet connectivity, we deployed the nationwide sensor network. The network has accommodate more than 100 station, and has two of dense installation. The application of this weather station network is for disaster protection/reduction/recovery and for educational material for various level of students.

\section{References}

[1] Live E! Project, http://www. live-e.org/

[2] Shinichi Doui, Satoshi Matsuura, Hideki Sunahara: An Infrastructure for Environmental Information Gathered by Fixedpoint and Mobile Sensors, DICOMO2006, IPSJ Symposium Series Vol.2006, No.6, $801-804$.

[3] Hiroki Ishizuka, Kenji Sasaki, Satoshi Matsuura, Makoto Kamiya, Hideki Sunahara, Hiroshi Esaki: Collecting Adaptive Data for Isolated Wireless Sensors with Patrol Nodes in Live E!, Proceeding of FMUIT2006, 249 - 253.

[4] Hideya Ochiai, Hiroshi Esaki: The Application of XML Translator for a Large-Scale Sensor Node Network, IEICE 2006, A-21-5.

[5] MATSUURA Satoshi and FUJIKAWA Kazutoshi and SUNAHARA Hideki: Mill: Scalable Area Management for P2P Network based on Geographical Location, Proceedings Euromedia 2006, $46-52$. 